\title{
Design of the Adaptive ship course control based on golden section method
}

\author{
Jinqiang Tong, Yang Liu \\ Marine college of Shandong jiaotong university, Weihai, Shandong,China
}

\begin{abstract}
Keywords: Ship steering controller Jury stability criterion; Golden; golden-section adaptive control of minimum variance control
\end{abstract}

\begin{abstract}
Research on ship motion control as the main design issues, the controller design is a vital part of the ship. in modern society, the ship sailing economy, safety and comfort controller has an important relationship with ship. many researchers at home and abroad have been unremitting efforts to improve the ship maneuverability. Adaptive control system during the boot process, How to ensure that the closed-loop system stability and dynamic quality is a very important issue. golden-section adaptive control is mainly for this type of problem. it is the golden ratio and minimum variance combined with the feedback control strategy.
\end{abstract}

\section{Introduction}

In the development and utilization of marine resources at the same time, ships as the main means of transport by sea also becoming larger and faster direction. However, with increasing traffic density on the sea, sailing, they also increasingly threatened. In navigation, how to use technology, developing high intelligence ship, is an important topic in today's major shipping countries.

When the ship sailing in the sea of time, is vulnerable to such waves, disturbances of the sea breezes and currents of the ocean environment. Therefore, it will roll, pitch and yaw changes. But on the whole, disturbance effects can be divided into two categories: is when the vessel's movement, the other is a swaying movement of the hull itself. Called the vessel movement is when you ship, operator with control devices, to change or to maintain the motion of the ship. And the oscillating movement of the hull itself, refers to the marine environment in reciprocating motion when disturbed.

Ship movements can be approximated as a second order systems with uncertain parameters and adaptive control method for this type of system can be used. Due to Adaptive control system startup when the closed-loop system stability and dynamic quality is very important. Based on this, the proposed Golden-section adaptive control solution. It is the golden ratio and minimum variance combined with the feedback control of feedback control strategy. When compared to other methods, this method is simple in design, convenient, robust, smooth the transition process, the advantages of easy to implement. The Jury stability criterion, could prove Golden and minimum variance combined with the feedback control strategy for stability for uncertain parameters feasible.

\section{Status of ship course control technology}

Since the since the invention of the magnetic compass, national experts and scholars has been committed to the research of ship automatic control and the stability of the system. Autopilot is developed on the basis of the rudder of a automatic steering control unit, ship course control system which is the most critical equipment, mainly for the course change control, autopilot control and track-keeping control.

Ship heading automatically rudder of development General is divided into four a stage, first to dates back to in the 1920 of the 20th century of mechanical type automatically rudder, was called first generation automatically rudder; to has in the 1950 of the 20th century with classical control theory of development produced has second generation automatically rudder) PID automatically rudder, in thereafter of for decades years in PID automatically rudder almost with in has all of ship Shang; 60 late with control theory and computer of rapid development, people proposed has can according to 
ship model parameter changes of since adapted rudder, Known as the third generation of the automatic steering gear; nearly 20 years with modern control theory of continuous improvement and development of nonlinear control theory, has been rapid development of intelligent control theory, intelligent control and intelligent control combination control algorithm and control algorithm has been applied in the automatic steering control system, forming a fourth-generation intelligent autopilot-autopilot.

Early in in the early 1920 of the 20th century, people on began on ship heading control system do line had has research, design has in at compared advanced of automatically rudder, that mechanical type automatically rudder, mechanical type automatically rudder technology just simple of proportion control, at to avoid caused big of shocks, need put controller of control gain set of is low, so this automatically rudder only for precision requirements compared low of heading control situation.

In the 1960 of the 20th century, with the development and application of classical control theory and electronics, appeared set to control technology and electronics in one of the second generation autopilot, that PID autopilot. Compared with the previous mechanical autopilot PID autopilot has been greatly improved, but it also has disadvantages.

To has 70 generation, people began will since adapted control system application to ship manipulation system in the, compared has representative of is in 70 generation early, J.VAmerongen, people success development has model reference since adapted rudder, to 70 generation medium-term, K.J.Astromd, people research design has since correction since adapted rudder, identification device used has based on minimum variance method of since adapted identification device, great of enhanced has ship of since adapted capacity. Adaptive rudder loads can adapt and change of outside interference, you can automatically adjust the control gain coefficients to changes in different and improve the ship's stability and level of automation.

Near more than 10 years to with modern control theory of constantly perfect and the computer of rapid development, various new control algorithm also have was application to automatically rudder system in the, which compared typical of has: neural network control, fuzzy control, experts control, variable structure control, anti-footwork control,, due to various control algorithm are has himself of advantages and disadvantages, so will different control algorithm combined is control territories of a research direction: as PID and Lu Rod control combined, PID and neural network control combined, Backstepping variable structure control, and was therefore known as fourth-generation intelligent autopilot-autopilot, intelligent autopilot is still in the research stage, has a very broad research and applications.

\section{Marine autopilot control principle}

Due to different uses, complete variety, when navigating the environments are very different, so different ships of the manipulation task there is a big difference. Water sports ship is usually controlled by a servo, automatic steering control system is used to control the rudder of the ship's movement. Ships sailing in the automatic steering control system has three States, namely rudder and yaw control state and control state. Most of the ship's autopilot with rudder work and course work, track control work only in a very few ships use, is currently the researchers an important research area.

When you ship on the open sea, you can long press of a ship set course, and should let the autopilot heading control to achieve the course to follow the course set sail. Ship heading control system in automatic control state, the ship will be able to comply with SC, which not only can reduce the labor intensity, and the ship's yaw slow trip is shortened, which can save a lot of fuel, helmsman and ship navigation using automatically up to a working state. Ship manipulation personnel according to different sea, different sailing route of situation, need in automatically rudder control system Shang set a given heading, automatically rudder control system will put given heading. and ship Shang of electric compass feedback back of ship actual heading phase compared, will its heading errors transfer to automatically rudder of heading controller system in the, again through controller sent 
instruction to rudder machine, in heading automatically rudder control system in the, after kept of adjustment, until ship heading errors more near zero, then ship of actual heading on can reached given heading, Which is the automatic steering control system of a ship.

How can I ship exactly in the formulation of the voyage on the route, which requires autopilot track control. In track control system in the, ship manipulation personnel need first will ship of initial location and sailing route storage in ship heading system control Taichung, and through GPS or Beidou system received ship of location information and calculation out track deviation volume, then according to track control mode calculation out heading by need of amendment volume, again through controller put heading control volume into into fuck rudder instruction sent to rudder machine, makes ship gradually driving to scheduled route Shang. Figure 1 is a sketch of a model ship track-keeping control,

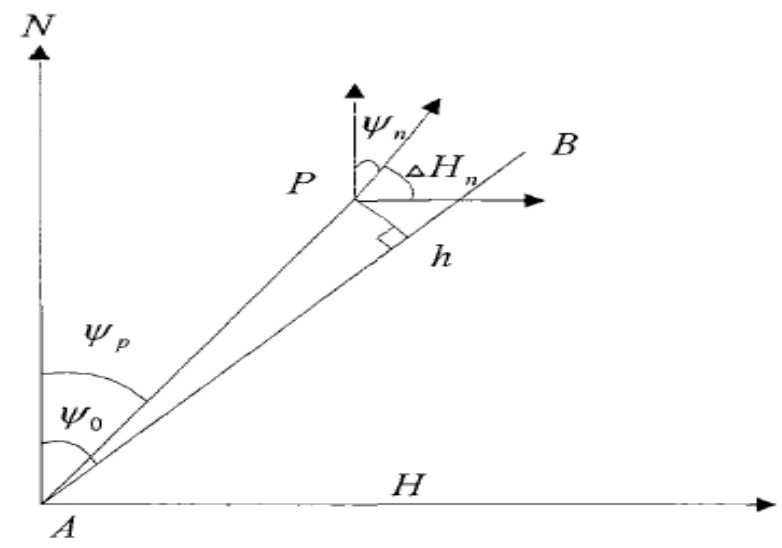

Fig .1 Ship track-keeping control system diagram

Ship heading control system includes the course change and course keeping in two ways, when sailing ships follow a set course, this is the course keeping problem. Dang set of heading need occurred change, this on need through playing rudder to change heading, this is ship heading track problem; in former of heading control system in the hope ship in by model parameter changes and outside disturbance Shi can as soon as possible of returned to set of heading Shang, which requirements ship can to shortest of regulation time, minimum of Super adjustable in guarantee ship stable of situation Xia fast track new set of heading.

\section{Ship steering controller design based on golden section method}

In order to effectively control control for different objects and different control requirements, proposes a variety of design theory and method of the controller, such as PID control method, control method of pole placement, minimum variance control, adaptive control and intelligent control methods, control theory and method for automatic control of the whole theory plays a large role in the development and the application.

Theory and method of the control, the controller design based on essential may be divided into two categories: the first based on a precise kinetic model, designed according to the requirements and control theory with analytical solutions for controller are not dependent on the exact model of the second kind, based on people's experience and control rules, artificially constructed by commissioning the controller or line identification and adjustment to achieve effective control.

Ship movements can be approximated as a second order systems with uncertain parameters and adaptive control method for this type of system can be used. And the golden-section adaptive control methods is the Golden and minimum variance combined with the feedback control strategy. Golden controller design method and stability of closed-loop systems, and golden-section Adaptive controller for closed-loop system stability in the parameters. Course track curves, and curve control the rudder as shown in Figure 2. 


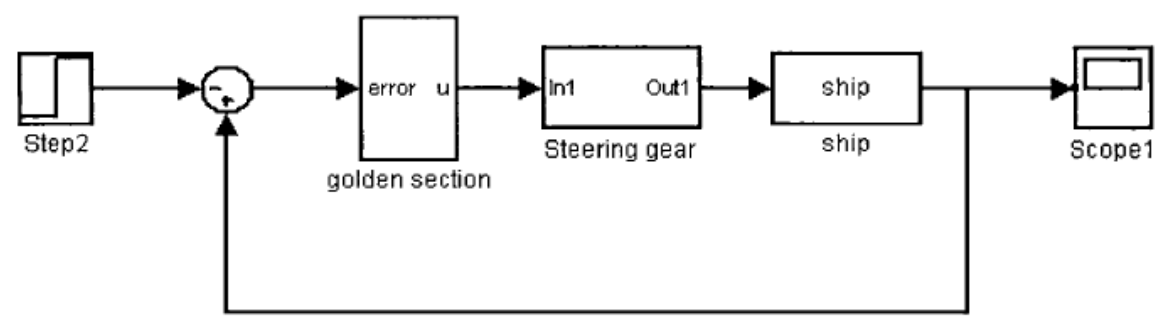

Fig .2 Ship heading control system simulation

Both types of controller design method of complex Liu Liu as it is not only the design is simple, convenient and high-performance. Especially for a kind of order and steady or slow time-varying complex objects with unknown parameters, when the field trial is not allowed when the method encountered difficulties. A case study in Adaptive control, system startup stage, for the first time, objects with unknown parameters, adaptive control cannot guarantee that the system is stable in General, may not function properly. In response to these difficulties in this chapter presents the golden section method and prove its stability.

Study on ship course control system designs, need to expect a reference heading sail. continuous are differentiable, but for actual reference signal it is impossible, so hope to use a desired signal continuous smooth sail, heading reference instead of an actual ship, a general and simple approach using model reference technique.

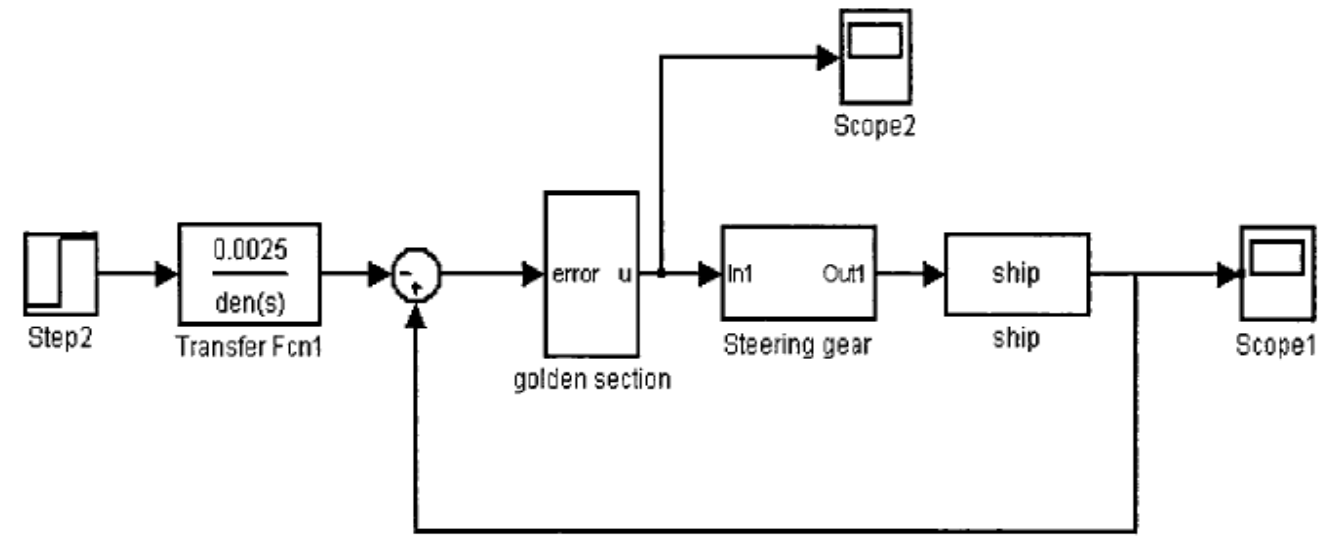

Fig .3 Heading control with reference filter simulation

After experiments with Golden controller is valid. Large model parameter perturbation and distractions exist with golden-section adaptive control simulation, in order to control, also using NCD parameters optimization of PID control in the same environment is simulated. Through experiment results can see, in no outside interference and parameter photo moving of situation Xia two species controller of control effect difference is unlikely to, even PID controller of rudder angle more small, effect better; but exists parameter photo moving and outside interference Xia, PID control effect quickly variable poor, appeared big of Super adjustable, control rudder angle changes also compared big, and gold segmentation since adapted control effect to obviously good Yu PID control. Shows the golden section law can deal with systems with unknown parameters, is very robust.

\section{References}

[1]Nollet F, FloquetT, pemiquetti W Observer based second order sliding mode control laws for step Permotors. Control Engineering Praetiee, 2008,25(6):420-450P.

[2]Nikoobin A,Haqhiqhi R. Lyapunov-based nonlinear disturbance observer for scrialn-Link robot maniPulators. J of Intelligent and Robotic Systems. Theory and APPlieations,2009, 10(5):120-160P

[3]Boroujeni,Elham,Amini. Adaptive sliding mode control for roll motions of ships, International Conference on Control, Automation and Systems, ICCAS,2008 
[4]Zhenghuayao, Guoyi, Huangxuewu. Investigation of Integrated Electric ProPulsion Simulation System of Modern Ship.IMECE，2003:102-125

[5]Donal Heffeman;Gabriel Leen.A time-triggered control network for industrial automation. Assembly Automation,2002.22(1):9-60

[6]Ronald G.Landman. Design and Analysis of CAN Networks for Vehieles[A], SAE 2000-01-2585

[7]Richard Piggin;Ken Young;Richard McLaughlin.The current fieldbus standards situation-a European view.Assembly automation,1999,19(4):286 